\title{
The dispersionless 2D Toda equation: dressing, Cauchy problem, longtime behaviour, implicit solutions and wave breaking
}

\author{
S. V. Manakov ${ }^{1, \S}$ and P. M. Santini ${ }^{2, \S}$ \\ ${ }^{1}$ Landau Institute for Theoretical Physics, Moscow, Russia \\ 2 Dipartimento di Fisica, Università di Roma "La Sapienza", and \\ Istituto Nazionale di Fisica Nucleare, Sezione di Roma 1 \\ Piazz.le Aldo Moro 2, I-00185 Roma, Italy \\ §e-mail: manakov@itp.ac.ru, paolo.santini@roma1.infn.it
}

September 14, 2021

\begin{abstract}
We have recently solved the inverse spectral problem for oneparameter families of vector fields, and used this result to construct the formal solution of the Cauchy problem for a class of integrable nonlinear partial differential equations in multidimensions, including the second heavenly equation of Plebanski and the dispersionless Kadomtsev - Petviashvili (dKP) equation, arising as commutation of vector fields. In this paper we make use of the above theory i) to construct the nonlinear Riemann-Hilbert dressing for the so-called two dimensional dispersionless Toda equation $(\exp (\varphi))_{t t}=\varphi_{\zeta_{1} \zeta_{2}}$, elucidating the spectral mechanism responsible for wave breaking; ii) we present the formal solution of the Cauchy problem for the wave form of it: $(\exp (\varphi))_{t t}=\varphi_{x x}+\varphi_{y y}$; iii) we obtain the longtime behaviour of the solutions of such a Cauchy problem, showing that it is essentially described by the longtime breaking formulae of the dKP solutions, confirming the expected universal character of the dKP equation as prototype model in the description of the gradient catastrophe of twodimensional waves; iv) we finally characterize a class of spectral data allowing one to linearize the $\mathrm{RH}$ problem, corresponding to a class of implicit solutions of the PDE.
\end{abstract}




\section{Introduction}

It was observed long ago [1] that the commutation of multidimensional vector fields can generate integrable nonlinear partial differential equations (PDEs) in arbitrary dimensions. Some of these equations are dispersionless (or quasiclassical) limits of integrable PDEs, having the dispersionless Kadomtsev - Petviashvili (dKP) equation $u_{x t}+u_{y y}+\left(u u_{x}\right)_{x}=0$ [2], 3] as universal prototype example, they arise in various problems of Mathematical Physics and are intensively studied in the recent literature (see, f.i., [4] - [18]). In particular, an elegant integration scheme applicable, in general, to nonlinear PDEs associated with Hamiltonian vector fields, was presented in [7] and a nonlinear $\bar{\partial}$ - dressing was developed in [13. Special classes of nontrivial solutions were also derived (see, f.i., [12, [15]).

The Inverse Spectral Transform (IST) for 1-parameter families of multidimensional vector fields has been developed in [19] (see also [20]). This theory, introducing interesting novelties with respect to the classical IST for soliton equations [21, 22], has allowed one to construct the formal solution

of the Cauchy problems for the second heavenly equation [23] in [19] and for the novel system of PDEs

$$
\begin{aligned}
& u_{x t}+u_{y y}+\left(u u_{x}\right)_{x}+v_{x} u_{x y}-v_{y} u_{x x}=0, \\
& v_{x t}+v_{y y}+u v_{x x}+v_{x} v_{x y}-v_{y} v_{x x}=0,
\end{aligned}
$$

in [24]. The Cauchy problem for the $v=0$ reduction of (1), the dKP equation, was also presented in [24], while the Cauchy problem for the $u=0$ reduction of (11), an integrable system introduced in [16], was given in [25]. This IST and its associated nonlinear Riemann - Hilbert (RH) dressing turn out to be efficient tools to study several properties of the solution space, such as: i) the characterization of a distinguished class of spectral data for which the associated nonlinear RH problem is linearized and solved, corresponding to a class of implicit solutions of the PDE (as it was done for the dKP equation in [26] and for the Dunajski generalization [27] of the second heavenly equation in [28]); ii) the construction of the longtime behaviour of the solutions of the Cauchy problem [26]; iii) the possibility to establish whether or not the lack of dispersive terms in the nonlinear PDE causes the breaking of localized initial profiles and, if yes, to investigate in a surprisingly explicit way the analytic aspects of such a wave breaking (as it was recently done for the $(2+1)$-dimensional dKP model in [26]). 
In this paper we make use of this theory to study another distinguished model arising as the commutation of vector fields, the so-called 2 dimensional dispersionless Toda (2ddT) equation

$$
\phi_{\zeta_{1} \zeta_{2}}=\left(e^{\phi_{t}}\right)_{t}, \quad \phi=\phi\left(\zeta_{1}, \zeta_{2}, t\right),
$$

or

$$
\varphi_{\zeta_{1} \zeta_{2}}=\left(e^{\varphi}\right)_{t t}, \quad \varphi=\phi_{t},
$$

also called Boyer-Finley [29] equation or $\mathrm{SU}(\infty)$ Toda equation [30], the natural continuous limit of the 2 dimensional Toda lattice [34, 35]

$$
\phi_{n \zeta_{1} \zeta_{2}}=c\left(e^{\phi_{n+1}-\phi_{n}}-e^{\phi_{n}-\phi_{n-1}}\right), \quad \phi=\phi_{n}\left(\zeta_{1}, \zeta_{2}\right) .
$$

The 2 ddT equation was probably first derived in 31 as an exact reduction of the second heavenly equation; then in [32] as a distinguished example of an integrable system in multidimensions. Some of its integrability properties have been investigated in [33] and the integration method presented in [7] is applicable to it. Both elliptic and hyperbolic versions of (2) are relevant, describing, for instance, integrable $\mathcal{H}$-spaces (heavens) [29, 36] and integrable Einstein - Weyl geometries [37]-38], 30]. String equations solutions [9] of it are relevant in the ideal Hele-Shaw problem [39, 40, 41, 42, 43.

The integrability of (2) follows from the fact that (2) is the condition of commutation $\left[\hat{L}_{1}, \hat{L}_{2}\right]=0$ for the following pair of one-parameter families of vector fields [8]:

$$
\begin{aligned}
& \hat{L}_{1}=\partial_{\zeta_{1}}+\lambda v \partial_{t}+\left(-\lambda v_{t}+\frac{\phi_{\zeta_{1} t}}{2}\right) \lambda \partial_{\lambda}, \\
& \hat{L}_{2}=\partial_{\zeta_{2}}+\lambda^{-1} v \partial_{t}+\left(\lambda^{-1} v_{t}-\frac{\phi_{\zeta_{2} t}}{2}\right) \lambda \partial_{\lambda},
\end{aligned}
$$

where

$$
v=e^{\frac{\phi_{t}}{2}}
$$

and $\lambda \in \mathbb{C}$ is the spectral parameter, implying the existence of common eigenfunctions of both vector fields; i.e., the existence of the Lax pair:

$$
\begin{aligned}
& \psi_{\zeta_{1}}=-\lambda v \psi_{t}+\left(\lambda v_{t}-\frac{\phi_{\zeta_{1} t}}{2}\right) \lambda \psi_{\lambda}, \\
& \psi_{\zeta_{2}}=-\lambda^{-1} v \psi_{t}-\left(\lambda^{-1} v_{t}-\frac{\phi_{\zeta_{2} t}}{2}\right) \lambda \psi_{\lambda} .
\end{aligned}
$$


Equations (7) and (2) can be written in the following "Hamiltonian" form [8]:

$$
\begin{aligned}
& \psi_{\zeta_{1}}+\left\{\mathcal{H}_{1}, \psi\right\}_{(\lambda, t)}=0, \quad \psi_{\zeta_{2}}+\left\{\mathcal{H}_{2}, \psi\right\}_{(\lambda, t)}=0, \\
& \mathcal{H}_{1 \zeta_{2}}-\mathcal{H}_{2 \zeta_{1}}-\left\{\mathcal{H}_{1}, \mathcal{H}_{2}\right\}_{(\lambda, t)}=0
\end{aligned}
$$

where

$$
\mathcal{H}_{1}=\lambda v-\frac{\phi_{\zeta_{1}}}{2}, \quad \mathcal{H}_{2}=-\lambda^{-1} v+\frac{\phi_{\zeta_{2}}}{2}
$$

and

$$
\{f, g\}_{(\lambda, t)}:=\lambda\left(f_{\lambda} g_{t}-f_{t} g_{\lambda}\right) .
$$

If, in particular,

$$
\zeta_{1}=z=\frac{x+i y}{2}, \quad \zeta_{2}=\bar{z}=\frac{x-i y}{2}, \quad x, y \in \mathbb{R},
$$

equation (2) becomes the following nonlinear wave equation

$$
\phi_{x x}+\phi_{y y}=\left(e^{\phi_{t}}\right)_{t},
$$

or

$$
\varphi_{x x}+\varphi_{y y}=\left(e^{\varphi}\right)_{t t} .
$$

In addition, if $|\lambda|=1$ and $\phi \in \mathbb{R}$, equations (9) give the "real" Hamiltonian formulation 32 ]

$$
\begin{aligned}
& \psi_{x}+\left\{H_{1}, \psi\right\}_{(\theta, t)}=0, \quad \psi_{y}+\left\{H_{2}, \psi\right\}_{(\theta, t)}=0, \\
& H_{1 y}-H_{2 x}-\left\{H_{1}, H_{2}\right\}_{(\theta, t)}=0,
\end{aligned}
$$

of (7) and (12), for the real Hamiltonians $H_{1}, H_{2}$ :

$$
\begin{aligned}
& H_{1}=\frac{i}{2}\left(\mathcal{H}_{1}+\mathcal{H}_{2}\right)=\sin \theta e^{\frac{\phi_{t}}{2}}-\frac{1}{2} \phi_{y}, \\
& H_{2}=\frac{1}{2}\left(\mathcal{H}_{2}-\mathcal{H}_{1}\right)=-\cos \theta e^{\frac{\phi_{t}}{2}}+\frac{1}{2} \phi_{x}
\end{aligned}
$$

and the Poisson bracket

$$
\{f, g\}_{(\theta, t)}:=f_{\theta} g_{t}-f_{t} g_{\theta},
$$

having introduced the parametrization

$$
\lambda=e^{-i \theta}, \quad \theta \in \mathbb{R} .
$$

The paper is organized as follows. In $\S 2$ we present the dressing scheme for equations (2) and (12), given in terms of a vector nonlinear RH problem. 
As for the dressing of dKP presented in [26], since the normalization of the eigenfunctions turns out to depend on the unknown solution of $2 \mathrm{ddT}$, a closure condition is necessary, allowing one to construct the solution of $2 \mathrm{ddT}$ through an implicit system of algebraic equations, whose inversion is responsible for the wave breaking of an initial localized profile. In $\S 3$ we present the IST for the 2ddT equation (12) and use it to obtain the formal solution of the Cauchy for such equation. In $\S 4$ we obtain the longtime behaviour of the solutions of such a Cauchy problem, showing that the solutions break also in the longtime regime, and that such regime is essentially described by the longtime breaking formulae of the dKP solutions [26], confirming the expected universal character of the dKP equation as prototype model in the description of the gradient catastrophe of two-dimensional waves. In $\S 5$ we characterize a class of $\mathrm{RH}$ spectral data allowing one to decouple and linearize the $\mathrm{RH}$ problem, generating a class of implicit solution of $2 \mathrm{ddT}$ parametrized by an arbitrary real function of one variable.

\section{Nonlinear RH dressing}

In this section we introduce the vector nonlinear $\mathrm{RH}$ problem enabling one to construct large classes of solutions of the Lax pair (7) and of the 2ddT equations (2) and (12).

Proposition. Consider the following vector nonlinear RH problem

$$
\xi_{j}^{+}(\lambda)=\xi_{j}^{-}(\lambda)+R_{j}\left(\xi_{1}^{-}(\lambda)+\nu_{1}(\lambda), \xi_{2}^{-}(\lambda)+\nu_{2}(\lambda)\right), \quad \lambda \in \Gamma, \quad j=1,2
$$

on an arbitrary closed contour $\Gamma$ of the complex $\lambda$ plane, where $\vec{R}(\vec{s})=$

$\left(R_{1}\left(s_{1}, s_{2}\right), R_{2}\left(s_{1}, s_{2}\right)\right)^{T}$ are given differentiable spectral data depending on the second argument $s_{2}$ through $\exp \left(i s_{2}\right)$ and satisfying the constraint

$$
\begin{aligned}
& \left\{\mathcal{R}_{1}\left(s_{1}, s_{2}\right), \mathcal{R}_{2}\left(s_{1}, s_{2}\right)\right\}_{\left(s_{1}, s_{2}\right)}=1, \\
& \mathcal{R}_{j}\left(s_{1}, s_{2}\right):=s_{j}+R_{j}\left(s_{1}, s_{2}\right), \quad j=1,2,
\end{aligned}
$$

with

$$
\{f, g\}_{\left(s_{1}, s_{2}\right)}:=f_{s_{1}} g_{s_{2}}-f_{s_{2}} g_{s_{1}}
$$

where $\nu_{j}, j=1,2$ are the explicit functions

$$
\vec{\nu}=\left(\begin{array}{c}
\nu_{1} \\
\nu_{2}
\end{array}\right)=\left(\begin{array}{c}
\left(\zeta_{1} \lambda+\zeta_{2} \lambda^{-1}\right) v-t-\zeta_{1} \phi_{\zeta_{1}} \\
i \ln \lambda+i \frac{\phi_{t}}{2}
\end{array}\right)
$$


and $\vec{\xi}^{+}=\left(\xi_{1}^{+}, \xi_{2}^{+}\right)^{T}$ and $\vec{\xi}^{-}=\left(\xi_{1}^{-}, \xi_{2}^{-}\right)^{T}$ are the unknown vector solutions of the RH problem (18), analytic rispectively inside and outside the contour $\Gamma$ and such that $\vec{\xi}^{-} \rightarrow \overrightarrow{0}$ as $\lambda \rightarrow \infty$. Then, assuming that the above $R H$ problem and its linearized form are uniquely solvable, we have the following results.

1) If

$$
\begin{aligned}
& \lim _{\substack{\lambda \rightarrow \infty \\
i \xi_{2}^{+}(0)}}\left(i \lambda \xi_{2}^{-}\right)=\phi_{t}, \\
& i \zeta_{1} e^{-\frac{\phi_{t}}{2}},
\end{aligned}
$$

it follows that $\vec{\pi}^{ \pm}=\vec{\xi}^{ \pm}+\vec{\nu}$ are common eigenfunctions of $\hat{L}_{1,2}: \hat{L}_{1} \vec{\pi}^{ \pm}=$ $\hat{L}_{2} \vec{\pi}^{ \pm}=\overrightarrow{0}$ satisfying the relations

$$
\left\{\pi_{1}^{ \pm}, \pi_{2}^{ \pm}\right\}_{(\lambda, t)}=\lambda\left(\pi_{1}^{ \pm} \pi_{2}^{ \pm}{ }_{t}-\pi_{1}^{ \pm} \pi_{1 \lambda}^{ \pm}\right)=i
$$

and the potentials $\phi_{\zeta_{1}}, \phi_{t}$, reconstructed through (22), solve the 2 ddT equation (2).

2) In addition, if the variables $\zeta_{1}, \zeta_{2}$ are specified as in (11), if the RH data satisfy the additional reality constraint

$$
\overrightarrow{\mathcal{R}}(\overline{\overrightarrow{\mathcal{R}}(\overline{\vec{s}})})=\vec{s}, \quad \forall \vec{s} \in \mathbb{C}
$$

and $\Gamma$ is the unit circle, then the eigenfunctions satisfy the following symmetry relation:

$$
\vec{\pi}^{-}(\lambda)=\overline{\vec{\pi}^{+}(1 / \bar{\lambda})}
$$

and $\phi \in \mathbb{R}$.

Remark 1 The RH problem (18) can obviously be formulated directly in terms of the eigenfunctions $\vec{\pi}^{ \pm}$as follows:

$$
\pi_{j}^{+}(\lambda)=\mathcal{R}_{j}\left(\pi_{1}^{-}(\lambda), \pi_{2}^{-}(\lambda)\right)=\pi_{j}^{-}(\lambda)+R_{j}\left(\pi_{1}^{-}(\lambda), \pi_{2}^{-}(\lambda)\right), \quad \lambda \in \Gamma, \quad j=1,2,
$$

with the normalization

$$
\vec{\pi}^{-}(\lambda)=\vec{\nu}(\lambda)+O\left(\lambda^{-1}\right),|\lambda|>>1
$$

Remark 2 The dependence of $\vec{R}$ on $s_{2}$ through $\exp \left(i s_{2}\right)$ ensures that the solutions $\vec{\xi}^{ \pm}$of the RH problem do not exhibit the $\ln \lambda$ singularity contained in the normalization (21). It follows that the eigenfunctions $\pi_{2}^{ \pm}$contain the 
$\ln \lambda$ singularity only as an additive singularity, while $\pi_{1}^{ \pm}$do not exhibit such singularity.

Remark 3 Before adding the closure conditions (22), the solutions $\vec{\xi}^{ \pm}$of the $\mathrm{RH}$ problem depend, via the normalization (21), on the undefined fields $\phi_{t}$ and $\phi_{\zeta_{1}}$, through the combination $\left(t+\zeta_{1} \phi_{\zeta_{1}}\right)$; then the two closure conditions (22) must be viewed as a nonlinear system of two algebraic equations for $\phi_{t}$ and $\phi_{\zeta_{1}}$ defining implicitly the solution $\phi_{\zeta_{1}}, \phi_{t}$ of the 2ddT equation. Therefore, as in the dKP case [26], we expect that this spectral features be responsible for the wave breaking of localized initial data evolving according to the nonlinear wave equation (3). Details on how two-dimensional waves evolving according to the $2 \mathrm{ddT}$ equation break will be presented elsewhere. An alternative closure, perhaps useful in the reality reduction case described in part 2) of the above Proposition, is given by the equations

$$
\begin{aligned}
& -i \frac{\xi_{1}^{+}(0)}{2}=\operatorname{Im}\left(t+z \phi_{z}\right), \\
& i \xi_{2}^{+}(0)=\phi_{t} .
\end{aligned}
$$

With this closure, indeed, we obtain a system of algebraic equations involving $t+z \phi_{z}$, its imaginary part and $\phi_{t}$.

Remark 4 The symmetry relations (25) are a distinguished example of the following symmetry of the common eigenfunctions of the Lax pair (7), when $\zeta_{1}=z, \zeta_{2}=\bar{z}$ as in (11) and $\phi \in \mathbb{R}$ :

if $\psi(\lambda)$ is a solution of (7), then $\overline{\psi(1 / \bar{\lambda})}$ is a solution too.

Remark 5 For part 2) of the above Proposition, when the contour $\Gamma$ is the unit circle, the RH problem is characterized by the following system of nonlinear integral equations for $\xi_{j}^{ \pm}(\lambda),|\lambda|=1$ (having parametrized $\lambda$ as in (17)):

$$
\begin{aligned}
& \xi_{j}^{ \pm}\left(e^{-i \theta}\right)-\frac{1}{2 \pi} \int_{0}^{2 \pi} \frac{d \theta^{\prime}}{1-(1 \mp \epsilon) e^{i\left(\theta^{\prime}-\theta\right)}} R_{j}\left(\left(z e^{-i \theta^{\prime}}+\bar{z} e^{i \theta^{\prime}}\right) v-t-z \phi_{z}+\xi_{1}^{-}\left(e^{-i \theta^{\prime}}\right),\right. \\
& \left.\theta^{\prime}+i \frac{\phi_{t}}{2}+\xi_{2}^{-}\left(e^{-i \theta^{\prime}}\right)\right)=0, \quad j=1,2
\end{aligned}
$$

and the closure conditions (22) read

$$
\begin{aligned}
& \phi_{z} e^{-\frac{\phi_{t}}{2}}=\frac{1}{2 \pi i} \int_{0}^{2 \pi} d \theta e^{-i \theta} R_{2}\left(\left(z e^{-i \theta}+\bar{z} e^{i \theta}\right) v-t-z \phi_{z}+\xi_{1}^{-}\left(e^{-i \theta}\right),\right. \\
& \left.\theta+i \frac{\phi_{t}}{2}+\xi_{2}^{-}\left(e^{-i \theta}\right)\right)=0
\end{aligned}
$$




$$
\begin{aligned}
& \phi_{t}=-\frac{1}{2 \pi i} \int_{0}^{2 \pi} d \theta R_{2}\left(\left(z e^{-i \theta}+\bar{z} e^{i \theta}\right) v-t-z \phi_{z}+\xi_{1}^{-}\left(e^{-i \theta}\right), \theta+\right. \\
& \left.i \frac{\phi_{t}}{2}+\xi_{2}^{-}\left(e^{-i \theta}\right)\right)=0 .
\end{aligned}
$$

Proof. For part 1), we first apply the operators $\hat{L}_{1,2}$ to the $\mathrm{RH}$ problem (26), obtaining the linearized RH problem $\hat{L}_{j} \vec{\pi}^{+}=A \hat{L}_{j} \vec{\pi}^{-}$, where $A$ is the Jacobian matrix of the transformation (26) : $A_{i j}=\partial \mathcal{R}_{i} / \partial s_{j}, i, j=1$, 2. Since, due to the normalization (27), $\hat{L}_{j} \vec{\pi}^{-} \rightarrow \overrightarrow{0}$ as $\lambda \rightarrow \infty$, it follows that, by uniqueness, $\vec{\pi}^{ \pm}$are common eigenfunctions of the vector fields $\hat{L}_{1,2}: \hat{L}_{1,2} \vec{\pi}^{ \pm}=\overrightarrow{0}$ and, consequently, that $\phi_{t}, \phi_{z}$ are solutions of the $2 \mathrm{ddT}$ equation (2). Then the eigenfunctions exhibit the following asymptotics:

$$
\begin{aligned}
& \pi_{1}^{-}=\lambda z v-t-z \phi_{z}+\lambda^{-1}\left(\bar{z} v+a^{-} v^{-1}\right)+O\left(\lambda^{-2}\right), \quad|\lambda|>>1, \\
& \pi_{1}^{+}=\lambda^{-1} \bar{z} v-t-\bar{z} \phi_{\bar{z}}+\lambda\left(z v+a^{+} v^{-1}\right)+O\left(\lambda^{2}\right), \quad|\lambda|<<1, \\
& \pi_{2}^{-}=i \ln \lambda+i \frac{\phi_{t}}{2}-i \lambda^{-1} \phi_{z} v^{-1}+O\left(\lambda^{-2}\right), \quad|\lambda|>>1 \\
& \pi_{2}^{+}=i \ln \lambda-i \frac{\phi_{t}}{2}+i \lambda \phi_{\bar{z}} v^{-1}+O\left(\lambda^{2}\right), \quad|\lambda|<<1,
\end{aligned}
$$

where

$$
a_{\bar{z}}^{-}=-a_{z}^{+}=\left(z e^{\phi_{t}}\right)_{z}-\left(\bar{z} e^{\phi_{t}}\right)_{\bar{z}} .
$$

implying the closure conditions (22),$(28)$. Since, from (26),$\left\{\pi_{1}^{+}, \pi_{2}^{+}\right\}_{(t, \lambda)}=$ $\left\{\mathcal{R}_{1}, \mathcal{R}_{2}\right\}_{\vec{\pi}^{-}}\left\{\pi_{1}^{-}, \pi_{2}^{-}\right\}_{(t, \lambda)}, \lambda \in \Gamma$, equation (19) implies that $\left\{\pi_{1}^{+}, \pi_{2}^{+}\right\}_{(t, \lambda)}=$ $\left\{\pi_{1}^{-}, \pi_{2}^{-}\right\}_{(t, \lambda)}, \lambda \in \Gamma$; i.e., the Poisson brackets of the \pm eigenfunctions are analytic in the whole complex $\lambda$ plane. Since $\left\{\pi_{1}^{-}, \pi_{2}^{-}\right\} \rightarrow i$ as $\lambda \rightarrow \infty$, it follows that $\left\{\pi_{1}^{+}, \pi_{2}^{+}\right\}=\left\{\pi_{1}^{-}, \pi_{2}^{-}\right\}=i$. For part 2), applying $\overrightarrow{\mathcal{R}}(\cdot)$ to the complex conjugate of the RH problem (26) and using the reality condition (24) it follows that $\overline{\vec{\pi}^{+}(\lambda)}=\vec{\pi}^{-}(\lambda),|\lambda|=1$. By the Schwartz reflection principle, it follows the symmetry (25) and, using the Lax pair (7), the reality condition $\phi \in \mathbb{R}$.

\section{The Cauchy problem for the 2ddT equation}

In this section we present the formal solution of the Cauchy problem for the wave form of the $2 \mathrm{ddT}$ equation:

$$
\begin{aligned}
& \left(e^{\phi_{t}}\right)_{t}=\phi_{x x}+\phi_{y y}, \quad x, y \in \mathbb{R}, \quad t>0, \quad \phi(x, y, t) \in \mathbb{R} \\
& \phi(x, y, 0)=A(x, y), \quad \phi_{t}(x, y, 0)=B(x, y) .
\end{aligned}
$$


where the assigned initial conditions $A(x, y), B(x, y)$ are localized in the $(x, y)$ plane for $x^{2}+y^{2} \rightarrow \infty$. To do it, we use the IST for vector fields developed in [19, 20, 24, 25].

In this respect, we recall two basic facts: since the Lax pair of the $2 \mathrm{ddT}$ is made of vector fields, $\mathrm{i}$ ) the space of eigenfunctions is a ring (if $f_{1}$ and $f_{2}$ are eigenfunctions, any differentiable function $F\left(f_{1}, f_{2}\right)$ is an eigenfunction); ii) since the vector fields are also Hamiltonian, the space of eigenfunctions is also a Lie algebra, whose Lie bracket is the Poisson bracket (10) (if $f_{1}$ and $f_{2}$ are eigenfunctions, also $\left\{f_{1}, f_{2}\right\}_{(\lambda, t)}$ is an eigenfunction).

Multiplying the first and second equations of (7) (with $\zeta_{1}=z, \zeta_{2}=\bar{z}$ as in (11)) by $\lambda^{-1}$ and $\lambda$ respectively, then adding and subtracting the resulting equations, one obtains the equivalent and more convenient Lax pair:

$$
\begin{array}{r}
\hat{\mathcal{L}}_{1} \psi:=\lambda \psi_{\bar{z}}-\lambda^{-1} \psi_{z}-\left(-2 v_{t}+\lambda \frac{\phi_{\bar{z} t}}{2}+\lambda^{-1} \frac{\phi_{z t}}{2}\right) \lambda \psi_{\lambda}=0, \\
\hat{\mathcal{L}}_{2} \psi:=\psi_{t}+\frac{v^{-1}}{2}\left(\lambda \psi_{\bar{z}}+\lambda^{-1} \psi_{z}\right)-\frac{v^{-1}}{4}\left(\lambda \phi_{\bar{z} t}-\lambda^{-1} \phi_{z t}\right) \lambda \psi_{\lambda}=0,
\end{array}
$$

where the first equation must be viewed as the spectral problem (in which $v_{t}$ shall be replaced, in the direct problem, by $\left(\phi_{z \bar{z}} / 2\right) \exp \left(-\phi_{t} / 2\right)$, due to (12)) and the second equation as $t$-evolution of the eigenfunction.

Eigenfunctions and spectral data. Now we introduce the Jost and analytic eigenfunctions for the spectral problem (35). Since the associated undressed operator: $\lambda \partial_{\bar{z}}-\lambda^{-1} \partial_{z}$ coincides with the undressed operator of the spectral problem for the $(2+1)$-dimensional self-dual Yang-Mills equation [44, the construction of the Jost and analytic Green's functions is taken from there.

We define Jost eigenfunctions of the spectral problem (35) on the unit circle of the complex $\lambda$ plane, using the parametrization (17). Introducing the convenient real variables $\xi, \eta, \theta^{\prime}$ as follows:

$$
\begin{aligned}
& \xi=\cos \theta x+\sin \theta y, \\
& \eta=-\sin \theta x+\cos \theta y, \\
& \theta^{\prime}=\theta,
\end{aligned}
$$

the Lax pair (35), (36) becomes

$$
\begin{gathered}
\hat{\mathcal{L}}_{1} \psi:=\psi_{\eta}-\frac{1}{2}\left[-\left(\phi_{\xi \xi}+\phi_{\eta \eta}\right) v^{-1}+\phi_{\xi t}\right]\left(\eta \psi_{\xi}-\xi \psi_{\eta}+\psi_{\theta^{\prime}}\right)=0, \\
\hat{\mathcal{L}}_{2} \psi:=\psi_{t}+v^{-1} \psi_{\xi}-\left(v^{-1}\right)_{\eta}\left(\eta \psi_{\xi}-\xi \psi_{\eta}+\psi_{\theta^{\prime}}\right)=0 .
\end{gathered}
$$


A convenient basis of Jost eigenfunctions are the solutions $f_{1}$ and $f_{2}$ of equation (38) satisfying the boundary conditions

$$
\vec{f}\left(\xi, \eta, \theta^{\prime}\right):=\left(\begin{array}{c}
f_{1}\left(\xi, \eta, \theta^{\prime}\right) \\
f_{2}\left(\xi, \eta, \theta^{\prime}\right)
\end{array}\right) \rightarrow\left(\begin{array}{c}
\xi \\
\theta^{\prime}
\end{array}\right), \text { as } \eta \rightarrow-\infty
$$

they are characterized by the linear integral equation

$$
\vec{f}=\left(\begin{array}{c}
\xi \\
\theta^{\prime}
\end{array}\right)+\frac{1}{2} \int_{-\infty}^{\eta} d \eta^{\prime}\left[-\left(\phi_{\xi \xi}+\phi_{\eta^{\prime} \eta^{\prime}}\right) v^{-1}+\phi_{\xi t}\right]\left(\eta^{\prime} \vec{f}_{\xi}-\xi \vec{f}_{\eta^{\prime}}+\vec{f}_{\theta^{\prime}}\right) .
$$

It follows that $f_{1}(\xi, \eta, \theta)$ and $f_{2}(\xi, \eta, \theta)-\theta$ are $2 \pi$-periodic in $\theta$.

The $\eta \rightarrow \infty$ limit of $\vec{f}$ defines the scattering vector $\vec{\sigma}(\xi, \theta)=\left(\sigma_{1}(\xi, \theta), \sigma_{2}(\xi, \theta)\right)^{T}$ as follows

$$
\vec{f}(\xi, \eta, \theta) \rightarrow \overrightarrow{\mathcal{S}}(\xi, \theta)=\left(\begin{array}{c}
\xi \\
\theta
\end{array}\right)+\vec{\sigma}(\xi, \theta), \text { as } \quad \eta \rightarrow \infty
$$

namely:

$$
\vec{\sigma}(\xi, \theta)=\frac{1}{2} \int_{\mathbb{R}} d \eta\left[-\left(\phi_{\xi \xi}+\phi_{\eta \eta}\right) v^{-1}+\phi_{\xi t}\right]\left(\eta \overrightarrow{f_{\xi}}-\xi \overrightarrow{f_{\eta}}+\overrightarrow{f_{\theta}}\right) .
$$

Also the scattering vector is $2 \pi$-periodic in $\theta: \vec{\sigma}(\xi, \theta+2 \pi)=\vec{\sigma}(\xi, \theta)$; i.e., its dependence on the second argument $\theta$ is through $\exp (i \theta)$.

The analytic eigenfunctions of (35) are defined instead via the integral equations:

$$
\begin{aligned}
& \vec{\psi}^{ \pm}(z, \bar{z}, \lambda)=\left(\begin{array}{c}
\psi_{1}^{ \pm}(z, \bar{z}, \lambda) \\
\psi_{2}^{ \pm}(z, \bar{z}, \lambda)
\end{array}\right)=\left(\begin{array}{c}
\lambda z+\lambda^{-1} \bar{z} \\
i \ln \lambda
\end{array}\right)+ \\
& \frac{i}{4} \int_{\mathbb{C}} d z^{\prime} \wedge d \bar{z}^{\prime} G^{ \pm}\left(z-z^{\prime}, \bar{z}-\bar{z}^{\prime}, \lambda\right)\left[\begin{array}{c}
\left.\phi_{z \bar{z}} v^{-1}+\lambda \frac{\phi_{\bar{z}^{\prime} t}}{2}+\lambda^{-1} \frac{\phi_{z^{\prime} t}}{2}\right] \lambda \vec{\psi}_{\lambda}^{ \pm}\left(z^{\prime}, \bar{z}^{\prime}, \lambda\right)
\end{array}\right.
\end{aligned}
$$

where $G^{ \pm}$are the analytic Green's functions

$$
G^{ \pm}(z, \bar{z}, \lambda)=\mp \frac{1}{\pi} \frac{1}{\left(\lambda z+\lambda^{-1} \bar{z}\right)}, \quad \operatorname{sgn}(1-|\lambda|)= \pm 1
$$

such that $\lambda G_{\bar{z}}^{ \pm}-\lambda^{-1} G_{z}^{ \pm}=\delta(z)$, reducing, on the unit circle $|\lambda|=1$, to

$$
G^{ \pm}(z, \bar{z}, \lambda)=\mp \frac{1}{\pi} \frac{1}{\xi \mp i \epsilon \eta}, \quad 0<\epsilon<<1 .
$$


Since $G^{+}$and $G^{-}$are analytic respectively inside and outside the unit circle of the complex $\lambda$ plane, then $\left(\psi_{1}^{+}, \psi_{2}^{+}\right)$and $\left(\psi_{1}^{-}, \psi_{2}^{-}\right)$are also analytic, respectively, inside and outside the unit circle of the complex $\lambda$ plane, after subtracting their singular parts, given respectively by $\left(\lambda^{-1} \bar{z} v, i \ln \lambda\right)$ and $(\lambda z v, i \ln \lambda)$, as it can be seen by solving the integral equations (44) by iteration or from the following $\lambda$ - asymptotics:

$$
\begin{aligned}
& \psi_{1}^{-}=\lambda z v-z \phi_{z}+\lambda^{-1}\left(\bar{z} v+a^{-} v^{-1}\right)+O\left(\lambda^{-2}\right), \quad|\lambda|>>1, \\
& \psi_{1}^{+}=\lambda^{-1} \bar{z} v-\bar{z} \phi_{\bar{z}}+\lambda\left(z v+a^{+} v^{-1}\right)+O\left(\lambda^{2}\right), \quad|\lambda|<<1, \\
& \psi_{2}^{-}=i \ln \lambda+i \frac{\phi_{t}}{2}-i \lambda^{-1} \phi_{z} v^{-1}+O\left(\lambda^{-2}\right), \quad|\lambda|>>1, \\
& \psi_{2}^{+}=i \ln \lambda-i \frac{\phi_{t}}{2}+i \lambda \phi_{\bar{z}} v^{-1}+O\left(\lambda^{2}\right), \quad|\lambda|<<1,
\end{aligned}
$$

where $a^{ \pm}$are defined in (33).

In addition, equations (46) imply the limits

$$
\begin{aligned}
& G^{+}\left(z-z^{\prime}, \bar{z}-\bar{z}^{\prime}, \lambda\right) \rightarrow-\frac{1}{\pi} \frac{1}{\xi-\xi^{\prime} \pm i \varepsilon}, \quad \text { as } \eta \rightarrow \mp \infty \\
& G^{-}\left(z-z^{\prime}, \bar{z}-\bar{z}^{\prime}, \lambda\right) \rightarrow \frac{1}{\pi} \frac{1}{\xi-\xi^{\prime} \mp i \varepsilon}, \quad \text { as } \eta \rightarrow \mp \infty .
\end{aligned}
$$

Therefore, on the unit circle $|\lambda|=1$, the $\eta \rightarrow-\infty$ limit of $\left(\psi_{1}^{+}, \psi_{2}^{+}\right)$and $\left(\psi_{1}^{-}, \psi_{2}^{-}\right)$are analytic respectively in the upper and lower parts of the complex $\xi$ plane, while the $\eta \rightarrow \infty$ limit of $\left(\psi_{1}^{+}, \psi_{2}^{+}\right)$and $\left(\psi_{1}^{-}, \psi_{2}^{-}\right)$are analytic respectively in the lower and upper parts of the complex $\xi$ plane. This mechanism, first observed in [44], plays an important role in the IST for vector fields (see [19], [20], [24], [25]).

Since the Jost eigenfunctions $\vec{f}=\left(f_{1}, f_{2}\right)^{T}$ are a good basis in the space of eigenfunctions of the spectral problem (38) for $|\lambda|=1$, one can express the analytic eigenfunctions in terms of them through the following formulae, valid for $|\lambda|=1$ :

$$
\vec{\psi}^{ \pm}=\overrightarrow{\mathcal{K}}^{ \pm}(\vec{f})=\vec{f}+\vec{\chi}^{ \pm}\left(f_{1}, f_{2}\right),
$$

defining the spectral data $\vec{\chi}^{ \pm}$as differentiable functions of two arguments. In the $\eta \rightarrow-\infty$ limit, equations (49) reduce to

$$
\lim _{\eta \rightarrow-\infty} \vec{\psi}^{ \pm}-\left(\begin{array}{c}
\xi \\
\theta
\end{array}\right)=\vec{\chi}^{ \pm}(\xi, \theta)
$$

implying that i) $\vec{\chi}^{+}(\xi, \theta)$ and $\vec{\chi}^{-}(\xi, \theta)$ are analytic in the first variable $\xi$ respectively in the upper and lower half parts of the complex $\xi$ plane, and ii) $\vec{\chi}^{ \pm}(\xi, \theta)$ are $2 \pi$-periodic in $\theta: \vec{\chi}^{ \pm}(\xi, \theta+2 \pi)=\vec{\chi}^{ \pm}(\xi, \theta)$ (their dependence on the second argument $\theta$ is through $\exp (i \theta))$. 
At $\eta \rightarrow \infty$, equations (49) reduce to

$$
\lim _{\eta \rightarrow \infty} \vec{\psi}^{ \pm}-\left(\begin{array}{c}
\xi \\
\theta
\end{array}\right)=\vec{\sigma}+\vec{\chi}^{ \pm}\left(\xi+\sigma_{1}, \theta+\sigma_{2}\right) .
$$

Applying the operator $\int_{\mathbb{R}} d \xi \int_{0}^{2 \pi} \frac{d \theta}{2 \pi} e^{-i(\omega \xi+n \theta)} ., n \in \mathbb{Z}$ to equations (51) and using the above established analiticity properties in $\xi$ and the $2 \pi$-periodicity in $\theta$, we obtain the following linear integral equations connecting the (Fourier transforms of the) scattering data $\vec{\sigma}$ to the (Fourier transforms of the) spectral data $\vec{\chi}^{ \pm}$:

$$
\tilde{\vec{\chi}}^{ \pm}(\omega, n)+H( \pm \omega)\left(\tilde{\vec{\sigma}}(\omega, n)+\int_{\mathbb{R}} d \omega^{\prime} \sum_{n^{\prime}=-\infty}^{\infty} \tilde{\vec{\chi}}^{ \pm}\left(\omega^{\prime}, n^{\prime}\right) Q\left(\omega^{\prime}, n^{\prime}, \omega, n\right)\right)=\overrightarrow{0},
$$

where $H$ is the Heaviside step function and

$$
\begin{aligned}
& Q\left(\omega^{\prime}, n^{\prime}, \omega, n\right)=\int_{\mathbb{R}} \frac{d \xi}{2 \pi} \int_{0}^{2 \pi} \frac{d \theta}{2 \pi} e^{i\left(\xi\left(\omega^{\prime}-\omega\right)+\left(n^{\prime}-n\right) \theta\right)}\left(e^{i\left(\omega^{\prime} \sigma_{1}(\xi, \theta)+n^{\prime} \sigma_{2}(\xi, \theta)\right)}-1\right), \\
& \tilde{\vec{\chi}}^{ \pm}(\omega, n)=\int_{\mathbb{R}} d \xi \int_{0}^{2 \pi} \frac{d \theta}{2 \pi} e^{-i(\omega \xi+n \theta)} \vec{\chi}^{+}(\xi, \theta), \\
& \tilde{\vec{\sigma}}(\omega, n)=\int_{\mathbb{R}} d \xi \int_{0}^{2 \pi} \frac{d \theta}{2 \pi} e^{-i(\omega \xi+n \theta)} \vec{\sigma}^{+}(\xi, \theta) .
\end{aligned}
$$

At last, eliminating, from equations (49), the Jost eigenfunctions $\vec{f}$, one obtains, through algebraic manipulation, the following vector nonlinear $\mathrm{RH}$ problem on the unit circle of the complex $\lambda$ plane:

$$
\begin{aligned}
& \psi_{1}^{+}=\mathcal{R}_{1}\left(\psi_{1}^{-}, \psi_{2}^{-}\right)=\psi_{1}^{-}+R_{1}\left(\psi_{1}^{-}, \psi_{2}^{-}\right), \quad|\lambda|=1, \\
& \psi_{2}^{+}=\mathcal{R}_{2}\left(\psi_{1}^{-}, \psi_{2}^{-}\right)=\psi_{2}^{-}+R_{2}\left(\psi_{1}^{-}, \psi_{2}^{-}\right) .
\end{aligned}
$$

We remark that the $2 \pi$-periodicity properties of the scattering data $\vec{\chi}^{ \pm}(\xi, \theta)$ in the variable $\theta$ imply that the dependence of $\vec{R}$ on the second argument $s_{2}$ is also through $\exp \left(i s_{2}\right)$, to guaranty that the $\ln \lambda$ singularity is just an additive one for $\psi_{2}^{ \pm}$, and is absent for $\psi_{1}^{ \pm}$.

Recapitulating, in the direct problem, at $t=0$, we go from the initial conditions $\phi, \phi_{t}$ of the $2 \mathrm{ddT}$ equation to the initial scattering vector $\vec{\sigma}(\xi, \theta)$; from it we construct, through the linear integral equations (52), the scattering data $\vec{\chi}^{ \pm}(\xi, \theta)$ and, through algebraic manipulation, the $\mathrm{RH}$ spectral 
data $\vec{R}(\vec{s})=\left(R_{1}\left(s_{1}, s_{2}\right), R_{2}\left(s_{1}, s_{2}\right)\right)$. In the inverse problem, one gives the RH spectral data $\vec{R}(\vec{s})$ and reconstructs the vector solutions $\vec{\psi}^{ \pm}$of the RH problem (54), defined by the normalization:

$$
\vec{\psi}^{-}=\left(\begin{array}{c}
\left(z \lambda+\bar{z} \lambda^{-1}\right) e^{\frac{\phi_{t}}{2}}-z \phi_{z} \\
i \ln \lambda+i \frac{\phi_{t}}{2}
\end{array}\right)+\vec{O}\left(\lambda^{-1}\right), \quad|\lambda|>>1 .
$$

At last, the closure conditions

$$
\begin{aligned}
& \lim _{\lambda \rightarrow \infty} \lambda\left(i \psi_{2}^{-}+\ln \lambda\right)=\phi_{z} e^{-\frac{\phi_{t}}{2}}, \\
& \lim _{\lambda \rightarrow 0}\left(i \psi_{2}^{+}+\ln \lambda\right)=\frac{\phi_{t}}{2}
\end{aligned}
$$

consequences of the asymptotics (47), allow one to reconstruct the solution of the $2 \mathrm{ddT}$ equation through the solution of a system of two algebraic equations for $\phi_{t}$ and $\phi_{z}$.

Time evolution of the spectral data. To construct the $t$-evolution of the spectral data we observe that $\vec{f}$ and $\vec{\psi}^{ \pm}$, eigenfunctions of the spectral problem (35): $\hat{\mathcal{L}}_{1} \vec{f}=\hat{\mathcal{L}}_{1} \vec{\psi}^{ \pm}=\overrightarrow{0}$, are solutions of the following equations involving the second Lax operator $\hat{\mathcal{L}}_{2} \vec{f}=\hat{\mathcal{L}}_{2} \vec{\psi}^{ \pm}=(1,0)^{T}$, implying the following elementary time evolutions of the data:

$$
\begin{aligned}
& \vec{\sigma}(\xi, \theta, t)=\vec{\sigma}(\xi-t, \theta, 0), \quad \vec{\chi}^{ \pm}(\xi, \theta, t)=\vec{\chi}^{ \pm}(\xi-t, \theta, 0), \\
& \vec{R}(\xi, \theta, t)=\vec{R}(\xi-t, \theta, 0) .
\end{aligned}
$$

In addition, it follows that the common Jost eigenfunctions $\vec{J}$ and the common analytic eigenfunctions $\vec{\pi}^{ \pm}$of the Lax pair (35), (36) are obtained from $\vec{f}$ and $\vec{\psi}^{ \pm}$simply as follows:

$$
\begin{aligned}
& \vec{J}:=\vec{f}-t(1,0)^{T}, \\
& \vec{\pi}^{ \pm}:=\vec{\psi}^{ \pm}-t(1,0)^{T} .
\end{aligned}
$$

It is easy to verify that the analytic eigenfunctions $\vec{\pi}^{ \pm}$, the RH data $\vec{R}(\vec{s})$ and the associated $\mathrm{RH}$ problem of this section coincide with those appearing in the dressing construction of $\S 2$.

Hamiltonian constraints on the data. The Hamiltonian character of the 2ddT dynamics implies the following formulae for the Poisson brackets of the relevant eigenfunctions:

$$
\left\{J_{1}, J_{2}\right\}_{(\lambda, t)}=\left\{\pi_{1}^{ \pm}, \pi_{2}^{ \pm}\right\}_{(\lambda, t)}=i
$$


which, in turn, imply that the transformations $\vec{s} \rightarrow \overrightarrow{\mathcal{K}}^{ \pm}(\vec{s})$ and $\vec{s} \rightarrow \overrightarrow{\mathcal{R}}(\vec{s})$ are canonical:

$$
\left\{\mathcal{K}_{1}^{ \pm}, \mathcal{K}_{2}^{ \pm}\right\}_{\left(s_{1}, s_{2}\right)}=\left\{\mathcal{R}_{1}, \mathcal{R}_{2}\right\}_{\left(s_{1}, s_{2}\right)}=1
$$

To prove (59), one first shows that $J_{3}:=\left\{J_{1}, J_{2}\right\}_{(\lambda, t)} \rightarrow i$ as $\eta \rightarrow-\infty$, $\pi_{3}^{-}:=\left\{\pi_{1}^{-}, \pi_{2}^{-}\right\}_{(\lambda, t)} \rightarrow i$ as $\lambda \rightarrow \infty, \pi_{3}^{+}:=\left\{\pi_{1}^{+}, \pi_{2}^{+}\right\}_{(\lambda, t)} \rightarrow i$ as $\lambda \rightarrow 0$. Since the vector fields are Hamiltonian, $J_{3}, \pi_{3}^{ \pm}$are also common eigenfunctions, and equations (59) hold, by uniqueness. Equations (60) are consequences of (59) and of the relations

$$
\vec{\pi}^{ \pm}=\overrightarrow{\mathcal{K}}^{ \pm}(\vec{J}), \quad \vec{\pi}^{+}=\overrightarrow{\mathcal{R}}\left(\vec{\pi}^{-}\right)
$$

Reality constraints. The definition $(58 \mathrm{~b})$ and the condition $\phi \in \mathbb{R}$ imply the symmetry relations

$$
\vec{\pi}^{-}(\lambda)=\overline{\vec{\pi}^{+}(1 / \bar{\lambda})}
$$

consequently, from (18), the reality constraint (24) on the RH data holds true.

Small field limit and Radon Transform. As for the IST of the heavenly [20] and dKP [24] equations, in the small field limit $|\phi|,\left|\phi_{t}\right|<<1$, the direct and inverse spectral transforms presented in this section reduce to the direct and inverse Radon transform [45]. Indeed, the mapping from the initial data $\{A(x, y), B(x, y)\}$ to the scattering vector $\vec{\sigma}$ reduces to the direct Radon transform:

$$
\begin{aligned}
& \vec{\sigma}(\xi, \theta) \sim \frac{1}{2} \int_{\mathbb{R}}\left(\begin{array}{c}
\eta \\
1
\end{array}\right)\left[-\left(\partial_{\xi}^{2}+\partial_{\eta}^{2}\right) A(x(\xi, \eta, \theta), y(\xi, \eta, \theta))+\right. \\
& \left.\partial_{\xi} B(x(\xi, \eta, \theta), y(\xi, \eta, \theta))\right] d \eta, \\
& x(\xi, \eta, \theta)=\xi \cos \theta-\eta \sin \theta, \quad y(\xi, \eta, \theta)=\xi \sin \theta+\eta \cos \theta,
\end{aligned}
$$

while the spectral data $\vec{\chi}^{ \pm}$and $\vec{R}$ are constructed from $\vec{\sigma}$ as follows:

$$
\vec{\chi}^{ \pm}(\xi, \theta) \sim-\hat{P}_{\xi}^{ \pm} \vec{\sigma}(\xi, \theta), \quad \vec{R}(\xi, \theta) \sim-i \hat{\mathcal{H}}_{\xi} \vec{\sigma}(\xi, \theta),
$$

where $\hat{P}_{\xi}^{ \pm}$and $\hat{\mathcal{H}}_{\xi}$ are rispectively the $( \pm)$ analyticity projectors and the Hilbert transform in the variable $\xi$ :

$$
\hat{P}_{\xi}^{ \pm} g(\xi):= \pm \frac{1}{2 \pi i} \int_{\mathbb{R}} \frac{d \xi^{\prime}}{\xi^{\prime}-(\xi \pm i 0)} g\left(\xi^{\prime}\right), \quad \hat{\mathcal{H}}_{\xi} g(\xi):=\frac{1}{\pi} P \int_{\mathbb{R}} \frac{d \xi^{\prime}}{\xi-\xi^{\prime}} g\left(\xi^{\prime}\right) .
$$


At last, the first of the closure conditions (56) of the inverse problem reduces to the inverse Radon transform

$$
\begin{aligned}
& \phi_{t}(x, y, t) \sim-\frac{1}{2 \pi i} \int_{0}^{2 \pi} d \theta R_{2}(\xi-t, \theta) \sim-\frac{1}{2 \pi^{2}} \int_{0}^{2 \pi} d \theta P \int_{\mathbb{R}} \frac{d \xi^{\prime}}{\xi^{\prime}-(\xi-t)} \sigma_{2}\left(\xi^{\prime}, \theta\right), \\
& \xi=x \cos \theta+y \sin \theta
\end{aligned}
$$

that can be shown to be equivalent to the well-known Poisson formula

$$
\begin{aligned}
& \phi(x, y, t)=\partial_{t} \int_{\mathbb{R}^{2}} \frac{d x^{\prime} d y^{\prime}}{2 \pi} L\left(x-x^{\prime}, y-y^{\prime}, t\right) A\left(x^{\prime}, y^{\prime}\right)+\int_{\mathbb{R}^{2}} \frac{d x^{\prime} d y^{\prime}}{2 \pi} L\left(x-x^{\prime},\right. \\
& \left.y-y^{\prime}, t\right) B\left(x^{\prime}, y^{\prime}\right)
\end{aligned}
$$

where

$$
L(x, y, t):=\frac{H\left(t^{2}-x^{2}-y^{2}\right)}{\sqrt{t^{2}-x^{2}-y^{2}}}
$$

and $H(\cdot)$ is the Heaviside step function, describing the solution of the Cauchy problem

$$
\begin{aligned}
& \phi_{t t}=\phi_{x x}+\phi_{y y}, \quad x, y \in \mathbb{R}, \quad t>0, \quad \phi(x, y, t) \in \mathbb{R} \\
& \phi(x, y, 0)=A(x, y), \quad \phi_{t}(x, y, 0)=B(x, y) .
\end{aligned}
$$

for the linear wave equation in $2+1$ dimensions.

\section{The longtime behaviour of the solutions}

In this section we show, as it was done in the dKP case [26], that the spectral mechanism causing the breaking of a localized initial condition evolving according to the $2 \mathrm{ddT}$ equation is present also in the longtime regime. We will actually show that the longtime breaking of the $2 d d T$ solutions is essentially described by the longtime breaking formulae of the dKP solutions found in [26]; this is an important confirmation of the expected universal character of the dKP equation as prototype model in the description of the gradient catastrophe of two-dimensional waves.

We remark that it is clearly meaningful to study the longtime behaviour of the solutions of the 2ddT equation only if no breaking takes place before, at finite time. In this section we assume that the initial condition be small, then the nonlinearity becomes important only in the longtime regime and no breaking takes place before. 
Motivated by the longtime behaviour of the solutions of the linear wave equation $u_{t t}=u_{x x}+u_{y y}$, localized, with amplitude $O\left(t^{-\frac{1}{2}}\right)$, in the region $\sqrt{x^{2}+y^{2}}-t=O(1)$, we study the longtime behaviour of the solutions of the $2 \mathrm{ddT}$ equation in the space-time region

$$
z=\frac{t+r}{2} e^{i \alpha}, \quad \alpha, r \in \mathbb{R}, \quad \alpha=O(1), \quad t>>1,
$$

implying that

$$
r=\sqrt{x^{2}+y^{2}}-t, \quad \alpha=\arctan \frac{y}{x} .
$$

Substituting (70) into the integral equations (29) and keeping in mind that, in the longtime regime, $\phi_{t}$ is small, so that, f. i., $v \sim 1+\phi_{t} / 2+\phi_{t}^{2} / 8$, we obtain

$$
\begin{aligned}
& \xi_{j}^{ \pm}(\lambda)-\frac{1}{2 \pi} \int_{0}^{2 \pi} \frac{d \theta^{\prime}}{1-(1 \mp \epsilon) e^{i\left(\theta^{\prime}-\theta\right)}} R_{j}\left(-2 t \sin ^{2}\left(\frac{\theta^{\prime}-\alpha}{2}\right)+r \cos \left(\theta^{\prime}-\alpha\right)+\right. \\
& \left.\frac{t+r}{2} \cos \left(\theta^{\prime}-\alpha\right) \phi_{t}\left(1+\frac{\phi_{t}}{4}\right)-z \phi_{z}+\xi_{1}^{-}\left(e^{-i \theta^{\prime}}\right), \theta^{\prime}+\xi_{2}^{-}\left(e^{-i \theta^{\prime}}\right)\right) \sim 0, \quad j=1,2 .
\end{aligned}
$$

Since the main contribution to these integrals occurs when $\sin \left(\left(\theta^{\prime}-\alpha\right) / 2\right) \sim 0$, we make the change of variable $\theta^{\prime}=\alpha-\mu^{\prime} / \sqrt{t}$, obtaining

$$
\begin{aligned}
& \xi_{j}^{ \pm}(\lambda)-\frac{1}{2 \pi \sqrt{t}} \int_{\mathbb{R}} \frac{d \mu^{\prime}}{1-(1 \mp \epsilon) e^{i\left(\alpha-\theta-\frac{\mu^{\prime}}{\sqrt{t}}\right)}} R_{j}\left(-\frac{\mu^{\prime 2}}{2}+X+\xi_{1}^{-}\left(e^{-i\left(\alpha-\frac{\mu^{\prime}}{\sqrt{t}}\right)}\right),\right. \\
& \left.\alpha+\xi_{2}^{-}\left(e^{-i\left(\alpha-\frac{\mu^{\prime}}{\sqrt{t}}\right)}\right)\right) \sim 0, \quad j=1,2
\end{aligned}
$$

where

$$
X:=r+\frac{t+r}{2} \phi_{t}+\frac{t}{8} \phi_{t}^{2}-z \phi_{z} .
$$

If $|\theta-\alpha|>>t^{-1 / 2}$, equations (73) imply that $\xi_{j}^{ \pm}(\lambda)=O\left(t^{-1 / 2}\right)$ :

$$
\begin{aligned}
& \xi_{j}^{ \pm}(\lambda) \sim \frac{1}{2 \pi \sqrt{t}\left(1-(1 \mp \epsilon) e^{i(\alpha-\theta)}\right)} \int_{\mathbb{R}} d \mu^{\prime} R_{j}\left(-\frac{\mu^{\prime 2}}{2}+X+\xi_{1}^{-}\left(e^{-i\left(\alpha-\frac{\mu^{\prime}}{\sqrt{t}}\right)}\right),\right. \\
& \left.\alpha+\xi_{2}^{-}\left(e^{-i\left(\alpha-\frac{\mu^{\prime}}{\sqrt{t}}\right)}\right)\right), \quad j=1,2 .
\end{aligned}
$$

If, instead, $\theta-\alpha=-\mu t^{-1 / 2},|\mu|=O(1)$, then $\xi_{j}^{ \pm}(\lambda)=O(1)$ :

$$
\begin{aligned}
& \xi_{j}^{ \pm}\left(e^{-i\left(\alpha-\frac{\mu}{\sqrt{t}}\right)}\right) \sim \frac{1}{2 \pi i} \int_{\mathbb{R}} \frac{d \mu^{\prime}}{\mu^{\prime}-(\mu \pm i \epsilon)} R_{j}\left(-\frac{\mu^{\prime 2}}{2}+X+\xi_{1}^{-}\left(e^{-i\left(\alpha-\frac{\mu^{\prime}}{\sqrt{t}}\right)}\right),\right. \\
& \left.\alpha+\xi_{2}^{-}\left(e^{-i\left(\alpha-\frac{\mu^{\prime}}{\sqrt{t}}\right)}\right)\right), \quad j=1,2 .
\end{aligned}
$$


Therefore it is not possible to neglect, in the above integral equations, $\xi_{j}^{-}, j=$ 1,2 in the arguments of $R_{j}, j=1,2$; it follows that these integral equations remain nonlinear even in the longtime regime.

At last, using equations (75), the asymptotic form of the closure conditions read, for $t>>1$ :

$$
\begin{aligned}
& \phi_{t} \sim-\frac{1}{2 \pi i \sqrt{t}} \int_{\mathbb{R}} d \mu^{\prime} R_{2}\left(-\frac{\mu^{\prime 2}}{2}+X+\xi_{1}^{-}\left(e^{-i\left(\alpha-\frac{\mu^{\prime}}{\sqrt{t}}\right)}\right),\right. \\
& \left.\alpha+\xi_{2}^{-}\left(e^{-i\left(\alpha-\frac{\mu^{\prime}}{\sqrt{t}}\right)}\right)\right), \\
& \phi_{z}=-e^{-i \alpha} \phi_{t}\left(1+\frac{\phi_{t}}{2}\right)\left(1+O\left(t^{-1}\right)\right) .
\end{aligned}
$$

Comparing (77) and (78), and using (70), we infer that $z \phi_{z} \sim-\frac{t+r}{2} \phi_{t}\left(1+\frac{\phi t}{2}\right)$. Using this asymptotic relation in (77), we finally obtain the following result.

In the space-time region

$$
\begin{aligned}
& z=\frac{t+r}{2} e^{i \alpha}, \quad \alpha, r \in \mathbb{R}, \quad t>>1, \\
& X:=r+(t+r) \phi_{t}+\frac{3 t}{8} \phi_{t}^{2}= \\
& \sqrt{x^{2}+y^{2}}-t+\sqrt{x^{2}+y^{2}} \phi_{t}+\frac{3 t}{8} \phi_{t}^{2}=O(1),
\end{aligned}
$$

the longtime $t>>1$ behaviour of the solutions of the $2 \mathrm{ddT}$ equation $\left(\exp \phi_{t}\right)_{t}=$ $\phi_{x x}+\phi_{y y}$ is described by the following implicit (scalar) equation:

$$
\phi_{t}=\frac{1}{\sqrt{t}} F\left(\sqrt{x^{2}+y^{2}}-t+\sqrt{x^{2}+y^{2}} \phi_{t}+\frac{3 t}{8} \phi_{t}^{2}, \arctan \frac{y}{x}\right)+o\left(\frac{1}{\sqrt{t}}\right),
$$

where $F$ is given by

$$
F(X, \alpha)=-\frac{1}{2 \pi i} \int_{\mathbb{R}} d \mu^{\prime} R_{2}\left(-\frac{\mu^{\prime 2}}{2}+X+a_{1}\left(\mu^{\prime} ; X, \alpha\right), \alpha+a_{2}\left(\mu^{\prime} ; X, \alpha\right)\right)
$$

and $a_{j}(\mu ; X, \alpha), j=1,2$ are the solutions of the integral equations

$$
\begin{aligned}
& a_{j}(\mu ; X, \alpha)=\frac{1}{2 \pi i} \int_{\mathbb{R}} \frac{d \mu^{\prime}}{\mu^{\prime}-(\mu-i \epsilon)} R_{j}\left(-\frac{\mu^{\prime 2}}{2}+X+a_{1}\left(\mu^{\prime} ; X, \alpha\right), \alpha+\right. \\
& \left.a_{2}\left(\mu^{\prime} ; X, \alpha\right)\right), \quad j=1,2 .
\end{aligned}
$$

Outside the asymptotic region (79) the solution decays faster.

We first remark that, since $\phi_{t}=O\left(t^{-\frac{1}{2}}\right)$, the condition $X=O(1)$ implies that $r=\sqrt{x^{2}+y^{2}}-t=O(\sqrt{t})$; it follows that, in the longtime regime $t>>$ 
1, the solution of the Cauchy problem for the $2 \mathrm{ddT}$ equation is concentrated, with amplitude $O\left(t^{-\frac{1}{2}}\right)$, in the asymptotic region $\sqrt{x^{2}+y^{2}}-t=O(\sqrt{t})$. We also remark that the asymptotic solution (80)-(82) is connected to the initial conditions of the Chauchy problem through the direct problem presented in the previous section.

\section{A distinguished class of implicit solutions}

In this section, in analogy with the results of [26], [28], we construct a class of explicit solutions of the vector nonlinear RH problem (18) and, correspondingly, a class of implicit solutions of the $2 \mathrm{ddT}$ equation parametrized by an arbitrary real spectral function of one variable.

Suppose that the two components of the RH spectral data $\vec{R}$ in (18) are given by:

$$
R_{j}\left(s_{1}, s_{2}\right)=(-1)^{j+1} \text { if }\left(e^{s_{1}+s_{2}}\right), \quad j=1,2,
$$

in terms of the single real spectral function $f$ of a single argument, depending on $s_{1}$ and $s_{2}$ only through their sum.

Then the RH problem (26) becomes

$$
\begin{aligned}
& \pi_{1}^{+}=\pi_{1}^{-}+i f\left(e^{\pi_{1}^{-}+\pi_{2}^{-}}\right), \quad|\lambda|=1, \\
& \pi_{2}^{+}=\pi_{2}^{-}-i f\left(e^{\pi_{1}^{-}+\pi_{2}^{-}}\right)
\end{aligned}
$$

and the following properties hold.

i) The reality and Hamiltonian constraints (24) and (19) are satisfied.

ii) $\pi_{1}^{+}+\pi_{2}^{+}=\pi_{1}^{-}+\pi_{2}^{-}$. Consequently, using the analyticity properties of the eigenfunctions, it follows that the functions $\Delta^{+}$and $\Delta^{-}$, defined by

$$
\Delta^{ \pm}:=\pi_{1}^{ \pm}+\pi_{2}^{ \pm}-\left(z \lambda+\bar{z} \lambda^{-1}\right) v-i \ln \lambda,
$$

are analytic respectively inside and outside the unit circle of the $\lambda$-plane and satisfy the equation $\Delta^{+}=\Delta^{-}$; therefore they are equal to a constant in $\lambda$. Evaluating such a constant at $\lambda=0$ and at $\lambda \rightarrow \infty$, we obtain the following equalities

$$
\Delta^{+}=\Delta^{-}=-t-\bar{z} \phi_{\bar{z}}-i \frac{\phi_{t}}{2}=-t-z \phi_{z}+i \frac{\phi_{t}}{2} .
$$


This implies that

i) the solutions of the $2 \mathrm{ddT}$ equation generated by the above $\mathrm{RH}$ problem satisfy the linear $(2+1)$-dimensional PDE

$$
\phi_{t}=i\left(\bar{z} \phi_{\bar{z}}-z \phi_{z}\right)
$$

and, substituting in (12) the expression of $\phi_{t}$ in terms of $\phi_{z}, \phi_{\bar{z}}$ given in (87), one obtains the following nonlinear two dimensional constraint:

$$
i\left(\bar{z} \partial_{\bar{z}}-z \partial_{z}\right)\left(e^{i\left(\bar{z} \phi_{\bar{z}}-z \phi_{z}\right)}\right)=\phi_{z \bar{z}}
$$

on the solutions of $2 \mathrm{ddT}$ constructed by the above $\mathrm{RH}$ problem.

ii) $\pi_{1}^{+}+\pi_{2}^{+}=\pi_{1}^{-}+\pi_{2}^{-}$is the following explicit and elementary function of $\lambda$ :

$$
w(\lambda):=\pi_{1}^{+}+\pi_{2}^{+}=\pi_{1}^{-}+\pi_{2}^{-}=\left(z \lambda+\bar{z} \lambda^{-1}\right) e^{-\frac{\phi_{t}}{2}}+i \ln \lambda-t-z \phi_{z}+i \frac{\phi_{t}}{2} .
$$

iii) Since, from (89),$\pi_{1}^{-}+\pi_{2}^{-}=w(\lambda)$ is an explicit function of $\lambda$, the vector nonlinear RH problem (84) decouples into two scalar, linear RH problems:

$$
\begin{aligned}
& \pi_{1}^{+}=\pi_{1}^{-}+i f\left(e^{w(\lambda)}\right), \\
& \pi_{2}^{+}=\pi_{2}^{-}-i f\left(e^{w(\lambda)}\right),
\end{aligned}
$$

whose explicit solutions are given by

$$
\xi_{j}^{ \pm}(\lambda)=(-1)^{j+1} \frac{1}{2 \pi i} \oint_{|\lambda|=1} \frac{d \lambda^{\prime}}{\lambda^{\prime}-(1 \mp \epsilon) e^{a r g \lambda}} f\left(e^{w\left(\lambda^{\prime}\right)}\right), \quad j=1,2,
$$

where $\xi_{j}^{ \pm}=\pi_{j}^{ \pm}-\nu_{j}$, and the closure conditions (22) read

$$
\phi_{z} e^{-\frac{\phi_{t}}{2}}=-\frac{1}{2 \pi i} \oint_{|\lambda|=1} d \lambda f\left(e^{w(\lambda)}\right), \quad \phi_{t}=\frac{1}{2 \pi i} \oint_{|\lambda|=1} \frac{d \lambda}{\lambda} f\left(e^{w(\lambda)}\right) .
$$

Although the RH problem (90) is linear, since $w(\lambda)$ in (89) depends on the unknowns $\phi_{t}, \phi_{z}$, the closure conditions (92) are a nonlinear algebraic system of two equations for the two unknowns $\phi_{t}, \phi_{z}$, defining implicitly a class of solutions of the $2 \mathrm{ddT}$ equation parametrized by the arbitrary real spectral function $f(\cdot)$ of a single variable.

Acknowledgements. This research has been supported by the RFBR grants 07-01-00446, 06-01-90840, and 06-01-92053, by the bilateral agreement between the Consortium Einstein and the RFBR, and by the bilateral agreement between the University of Roma "La Sapienza" and the Landau Institute for Theoretical Physics of the Russian Academy of Sciences. 


\section{References}

[1] V. E. Zakharov and A. B. Shabat, Functional Anal. Appl. 13, 166-174 (1979).

[2] R. Timman, "Unsteady motion in transonic flow", Symposium Transsonicum, Aachen 1962. Ed. K. Oswatitsch, Springer 394-401.

[3] E. A. Zobolotskaya and R. V. Kokhlov, "Quasi - plane waves in the nonlinear acoustics of confined beams", Sov. Phys. Acoust. 15, n. 1 (1969) $35-40$.

[4] Y. Kodama and J. Gibbons, "Integrability of the dispersionless KP hierarchy", Proc. 4th Workshop on Nonlinear and Turbulent Processes in Physics, World Scientific, Singapore 1990.

[5] B. Kupershmidt, J. Phys. A: Math. Gen. 23, 871 (1990).

[6] V. E. Zakharov, "Dispersionless limit of integrable systems in 2+1 dimensions", in Singular Limits of Dispersive Waves, edited by N.M.Ercolani et al., Plenum Press, New York, 1994.

[7] I. M. Krichever, "The $\tau$-function of the universal Witham hierarchy, matrix models and topological field theories", Comm. Pure Appl. Math. 47, 437-475 (1994).

[8] K. Takasaki and T. Takebe, arXiv:hep-th/9112042.

[9] K. Takasaki and T. Takebe, Rev. Math. Phys. 7, 743 (1995).

[10] M. Dunajski and L. J. Mason, "Hyper-Kähler hierachies and their twistor theory", Comm. Math. Phys. 213, 641-672 (2000). "Twistor theory of hyper-Kähler metrics with hidden symmetries", J. Math. Phys., 44, 3430-3454 (2003).

[11] M. Dunajski, L. J. Mason and P. Tod, "Einstein-Weyl geometry, the dKP equation and twistor theory", J. Geom. Phys. 37 63-93 (2001).

[12] M. Dunajski and K. P. Tod, "Einstein-Weyl spaces and dispersionless Kadomtsev-Petviashvili equation from Painlevé I and II"; arXiv:nlin.SI/0204043. 
[13] B. Konopelchenko, L. Martinez Alonso and O. Ragnisco, "The $\bar{\partial}-$ approach for the dispersionless KP hierarchy", J.Phys. A: Math. Gen. 34 10209-10217 (2001).

[14] L. Martinez Alonso and A. B. Shabat, "Towards a theory of differential constraints of a hydrodynamic hierarchy" J. Nonlinear Math. Phys 10(2) (2003) 229. A. B. Shabat and L. Martinez Alonso: "On the prolongation of a hierarchy of hydrodynamic chains", in New Trends in Integrability and Partial Solvability, Editors A.B. Shabat et al., Proceedings of the NATO Advanced Research Workshop (2002, Cadiz, Spain), NATO Sci. Ser. II Math. Phys. Chem., Vol. 132, Dordrecht, Kluwer Academic Publishers, 2004, 263-280. L. Martinez Alonso and A. B. Shabat: "Hydrodynamic reductions and solutions of a universal hierarchy", Theoret. and Math. Phys., 2004, V.140, 1073-1085.

[15] F. Guil, M. Manas and L. Martinez Alonso, "On twistor solutions of the dKP equation", J. Phys. A:Math. Gen. 36 (2003) 6457-6472.

[16] M. V. Pavlov: "Integrable hydrodynamic chains", J. Math. Phys. 44 (2003) 4134-4156.

[17] E. V. Ferapontov and K. R. Khusnutdinova: "On integrability of $(2+1)$ dimensional quasilinear systems", Comm. Math. Phys. 248 (2004) 187206.

[18] B. Konopelchenko and F. Magri, "Dispersionless integrable equations as coisotropic deformations. Extensions and reductions". arXiv:nlin/0608010.

[19] S. V. Manakov and P. M. Santini: "Inverse scattering problem for vector fields and the Cauchy problem for the heavenly equation", Physics Letters A 359 (2006) 613-619. http://arXiv:nlin.SI/0604017.

[20] S. V. Manakov and P. M. Santini: "Inverse scattering problem for vector fields and the heavenly equation"; http://arXiv:nlin.SI/0512043.

[21] V. E. Zakharov, S. V. Manakov, S. P. Novikov and L. P. Pitaevsky, Theory of solitons, Plenum Press, New York, 1984. 
[22] M. J. Ablowitz and P. A. Clarkson, Solitons, nonlinear evolution equations and Inverse Scattering, London Math. Society Lecture Note Series, vol. 194, Cambridge University Press, Cambridge (1991).

[23] J. F. Plebanski, "Some solutions of complex Einstein equations", J. Math. Phys. 16, 2395-2402 (1975).

[24] S. V. Manakov and P. M. Santini: "The Cauchy problem on the plane for the dispersionless Kadomtsev-Petviashvili equation"; JETP Letters, 83, No 10, 462-466 (2006). http://arXiv:nlin.SI/0604016.

[25] S. V. Manakov and P. M. Santini: Theor. Math. Phys. 152(1), 10041011 (2007).

[26] S. V. Manakov and P. M. Santini: "On the solutions of the dKP equation: the nonlinear Riemann-Hilbert problem, longtime behaviour, implicit solutions and wave breaking"; J. Phys. A: Math. Theor. 41 (2008) 055204 (23pp).

[27] M. Dunajski, Proc. Royal Soc. A 458, 1205.

[28] L. Bogdanov, V. Dryuma and S. V. Manakov: "Dunajski generalization of the second heavenly equation: dressing method and the hierarchy"; J. Phys. A: Math. Theor. 40 14383-14393, doi: 10.1088/1751$8113 / 40 / 48 / 005$.

[29] C. Boyer and J. D. Finley, "Killing vectors in self-dual, Euclidean Einstein spaces", J. Math. Phys. 23 (1982), 1126-1128.

[30] R. S. Ward, "Einstein-Weyl spaces and SU( $\infty)$ Toda fields", Class. Quantum Grav. 7 (1990) L95-L98.

[31] J. D. Finley and J. F. Plebanski: "The classification of all $\mathcal{K}$ spaces admitting a Killing vector", J. Math. Phys. 20, 1938 (1979).

[32] V. E. Zakharov: "Integrable systems in multidimensional spaces", Lecture Notes in Physics, Springer-Verlag, Berlin 153 (1982), 190-216.

[33] M. V. Saveliev, Commun. Math. Phys. 121 (1989), 283. M. V. Saveliev, Teoreticheskaya i Matematicheskaya Fisika, 92 No. 3, (1992) 457-465. 
[34] G. Darboux, Lecons sur la théorie générale des surfaces. II, Gauthier Villars, Paris 1888.

[35] A. V. Mikhailov, "On the Integrability of two-dimensional Generalization of the Toda Lattice", Letters in Journal of Experimental and Theoretical Physics, v.30, p. 443-448, 1979.

[36] J. D. Gegenberg and A. Das, "Stationary Riemaniann space-times with self-dual curvature", Gen. Rel. Grav. 16 (1984), 817-829.

[37] N. J. Hitchin, "Complex manifolds and Einstein's equations", in Twistor Geometry and Nonlinear Systems, H. D. Doebner and T. Weber (eds), Lecture Notes in Mathematics, vol. 970 (Springer-Verlag 1982).

[38] P. E. Jones and K. P. Tod, "Minitwistor spaces and Einstein-Weyl spaces", Class. Quantum Grav. 2 (1985), 565-577.

[39] M. Mineev-Weinstein, P. Wigmann and A. Zabrodin, Phys. Rev. Lett. 84, 5106 (2000).

[40] P. Wigmann and A. Zabrodin, Comm. Math. Phys. 213, 523 (2000).

[41] I. Krichever, A. Marshakov and A. Zabrodin, "Integrable structure of the Dirichlet boundary problem in multiply-connected domains". Comm. Math. Phys. 259 (2005), no. 1, 1-44.

[42] S.-Y. Lee, E. Bettelheim, P. Wiegmann, "Bubble break-off in Hele-Shaw flows-singularities and integrable structures" Phys. D 219 (2006), no. 1, $22-34$.

[43] L. Martinez Alonso and E. Medina: "Regularisation of Hele-Shaw flows, multiscaling expansions and the Painlevé I equation", arXiv:0710.3731.

[44] S. V. Manakov and V. E. Zakharov, "Three-dimensional model of relativistic-invariant field theory, integrable by the inverse scattering transform"; Letters in Mathematical Physics 5, 247-253 (1981).

[45] S. R. Deans, The Radon transform and some of it applications, second edition, Krieger, Malabar, FL, 1993. 